Russell A. Gordon, Department of Mathematics, Whitman College, Walla

Walla, WA 99362

\title{
AN ITERATED LIMITS THEOREM APPLIED TO THE HENSTOCK INTEGRAL
}

\begin{abstract}
An iterated limits theorem is used to establish two sets of necessary and sufficient conditions for a Henstock integral convergence theorem. The motivation for the iterated limits theorem originates from Bartle's notion of $\gamma$-convergence.
\end{abstract}

In a recent paper in this journal, Bartle [1] found necessary and sufficient conditions for a Henstock integral convergence theorem. The key concept (defined below) is the $\gamma$-convergence of a sequence $\left\{f_{k}\right\}$ of functions. In this paper, we examine some of the properties of $\gamma$-convergence, point out some difficulties with this concept, and suggest a minor modification to the definition of $\gamma$-convergence. With this modification, Bartle's theorem is actually a special case of a general convergence theorem involving iterated limits. In addition, this iterated limits theorem provides a second set of necessary and sufficient conditions for a Henstock integral convergence theorem.

We will assume that the reader is familiar with the notation and terminology associated with the Henstock integral. The symbol $S(f, P)$ will denote the Riemann sum of $f$ on the $\delta$-fine tagged partition $P$. The first step is to define the $\gamma$-convergence of a sequence $\left\{f_{k}\right\}$ of functions and the (somewhat related) concept of uniform Henstock integrability. This latter concept is the basis for the "best" (in some sense, see [2]) convergence theorem for the Henstock integral.

Definition 1 Let $\left\{f_{k}\right\}$ be a sequence of Henstock integrable functions defined on $[a, b]$ and let $f:[a, b] \rightarrow \mathbb{R}$.

(a) The sequence $\left\{f_{k}\right\}$ is $\gamma$-convergent to $f$ on $[a, b]$ if for each $\epsilon>0$ there exists a positive integer $N_{\epsilon}$ such that for each $k \geq N_{\epsilon}$ there exists a positive function $\delta_{k}$ defined on $[a, b]$ such that

$$
\left|S\left(f_{k}, P\right)-S(f, P)\right|<\epsilon
$$

Mathematical Reviews subject classification: 26A39

Received by the editors August 14, 1995 


\section{An Iterated Limits Theorem Applied to the Henstock Integral775}

whenever $P$ is a $\delta_{k}$-fine tagged partition of $[a, b]$.

(b) The sequence $\left\{f_{k}\right\}$ is uniformly Henstock integrable on $[a, b]$ if for each $\epsilon>0$ there exists a positive function $\delta$ defined on $[a, b]$ such that

$$
\left|S\left(f_{k}, P\right)-\int_{a}^{b} f_{k}\right|<\epsilon
$$

whenever $P$ is a $\delta$-fine tagged partition of $[a, b]$.

The first point to mention is that Bartle's definition of $\gamma$-convergence does not require the functions to be Henstock integrable. However, since this notion will only be applied to sequences of integrable functions, there is no harm in adding integrability to the definition.

These two concepts are not comparable in the sense that one implies the other. For each positive integer $k$, define

$$
f_{k}(x)=\left\{\begin{array}{ll}
k, & \text { if } 0 \leq x \leq 1 ; \\
0, & \text { otherwise }
\end{array} \quad \text { and } \quad g_{k}(x)= \begin{cases}k, & \text { if } x=0 \\
0, & \text { if } x \neq 0\end{cases}\right.
$$

The sequence $\left\{f_{k}\right\}$ is uniformly Henstock integrable on $[0,1]$, but it is not $\gamma$-convergent to any function $f$ defined on $[0,1]$. The sequence $\left\{g_{k}\right\}$ is $\gamma$-convergent on $[0,1]$ to the function $g$ defined by $g(x)=0$ for all $x$, but it is not uniformly Henstock integrable on $[0,1]$. These facts are not hard to justify and will primarily be left to the reader, but we will at least show that $\left\{f_{k}\right\}$ is not $\gamma$-convergent on $[0,1]$. Suppose that $\left\{f_{k}\right\}$ is $\gamma$-convergent to $f$ on $[0,1]$. Corresponding to $\epsilon=1$, choose $N_{1}>2$ as in the definition of $\gamma$-convergence. Suppose that $k>N_{1}$ and let $\delta=\min \left\{\delta_{k}, \delta_{2 k}\right\}$. If $P$ is a $\delta$-fine tagged partition of $[a, b]$, then

$k=\left|S\left(f_{k}, P\right)-S\left(f_{2 k}, P\right)\right| \leq\left|S\left(f_{k}, P\right)-S(f, P)\right|+\left|S(f, P)-S\left(f_{2 k}, P\right)\right|<2$,

a contradiction. It follows that $\left\{f_{k}\right\}$ is not $\gamma$-convergent on $[0,1]$.

The sequence $\left\{g_{k}\right\}$ reveals a potential problem with $\gamma$-convergence. For all of the common types of convergence, the limit is unique. This result fails dramatically for $\gamma$-convergence. The sequence $\left\{g_{k}\right\}$ is $\gamma$-convergent on $[0,1]$ to any Henstock integrable function $g$ with the property that $\int_{0}^{1} g=0$. The same would be true even if $g_{k}(x)=0$ for all $x$, so even a constant sequence does not have a unique limit. To verify this claim, let $\epsilon>0$ and fix a positive integer $k$. Choose a positive function $\delta$ on $[a, b]$ such that

$$
\begin{gathered}
\left|S\left(g_{k}, P\right)\right|=\left|S\left(g_{k}, P\right)-\int_{0}^{1} g_{k}\right|<\epsilon / 2 ; \\
|S(g, P)|=\left|S(g, P)-\int_{0}^{1} g\right|<\epsilon / 2 ;
\end{gathered}
$$


whenever $P$ is a $\delta$-fine tagged partition of $[0,1]$. For such $P$,

$$
\left|S\left(g_{k}, P\right)-S(g, P)\right| \leq\left|S\left(g_{k}, P\right)\right|+|S(g, P)|<\epsilon .
$$

Therefore, the sequence $\left\{g_{k}\right\}$ is $\gamma$-convergent to $g$ on $[0,1]$.

It seems to me that this amount of non-uniqueness is unacceptable. One potential method for resolving this difficulty is the following. Let $\left\{f_{k}\right\}$ be a sequence of functions defined on $[a, b]$ that is $\gamma$-convergent and let $\mathcal{F}$ be the collection of all functions $f:[a, b] \rightarrow \mathbb{R}$ such that $\left\{f_{k}\right\}$ is $\gamma$-convergent to $f$ on $[a, b]$. Select a function $f \in \mathcal{F}$ such that there is a subsequence of $\left\{f_{k}\right\}$ that converges pointwise almost everywhere on $[a, b]$ to $f$. This would, in this context at least, be a natural choice for $f$. However, the sequence $\{\sin (2 k \pi x)\}$ is $\gamma$-convergent to 0 on $[0,1]$, but this sequence contains no subsequences that converge pointwise almost everywhere on $[0,1]$. The bottom line of this discussion seems to be that pointwise convergence should be added as part of the definition of $\gamma$-convergence. Since integral convergence theorems almost always involve some type of convergence, often pointwise convergence (or what usually amounts to the same thing, pointwise convergence almost everywhere), there is not a great loss of generality to include this condition.

When pointwise convergence is included, a uniformly Henstock integrable sequence is also $\gamma$-convergent. This is the content of the next theorem.

Theorem 1 Let $\left\{f_{k}\right\}$ be a sequence of Henstock integrable functions defined on $[a, b]$, let $f$ be a function defined on $[a, b]$, and suppose that $\left\{f_{k}\right\}$ converges pointwise to $f$ on $[a, b]$. If $\left\{f_{k}\right\}$ is uniformly Henstock integrable on $[a, b]$, then $\left\{f_{k}\right\}$ is $\gamma$-convergent to $f$ on $[a, b]$.

Proof. It is not difficult to prove (see [2]) that the sequence $\left\{\int_{a}^{b} f_{k}\right\}$ is a Cauchy sequence. Let $\epsilon>0$. Choose a positive integer $N_{\epsilon}$ such that

$$
\left|\int_{a}^{b} f_{k}-\int_{a}^{b} f_{n}\right|<\epsilon / 4
$$

for all $k, n \geq N_{\epsilon}$. Since $\left\{f_{k}\right\}$ is uniformly Henstock integrable on $[a, b]$, there exists a positive function $\delta$ defined on $[a, b]$ such that

$$
\left|S\left(f_{k}, P\right)-\int_{a}^{b} f_{k}\right|<\epsilon / 4
$$

whenever $P$ is a $\delta$-fine tagged partition of $[a, b]$. (In other words, the choice of the function $\delta$ will be independent of $k \geq N_{\epsilon}$.) Fix $k \geq N_{\epsilon}$ and suppose that $P$ is a $\delta$-fine tagged partition of $[a, b]$. Since $\left\{f_{k}\right\}$ converges pointwise to $f$ on $[a, b]$, there exists a positive integer $n \geq N_{\epsilon}$ such that

$$
\left|S\left(f_{n}, P\right)-S(f, P)\right|<\epsilon / 4 \text {. }
$$


It follows that

$$
\begin{aligned}
\mid S\left(f_{k}, P\right) & -S(f, P) \mid \\
\leq & \left|S\left(f_{k}, P\right)-\int_{a}^{b} f_{k}\right|+\left|\int_{a}^{b} f_{k}-\int_{a}^{b} f_{n}\right|+\left|\int_{a}^{b} f_{n}-S\left(f_{n}, P\right)\right| \\
& +\left|S\left(f_{n}, P\right)-S(f, P)\right| \\
< & \epsilon / 4+\epsilon / 4+\epsilon / 4+\epsilon / 4=\epsilon .
\end{aligned}
$$

It follows that $\left\{f_{k}\right\}$ is $\gamma$-convergent to $f$ on $[a, b]$. This completes the proof. $\square$

To show that the converse is false, consider the following example. For each positive integer $k$, define $f_{k}:[0,3] \rightarrow \mathbb{R}$ by

$$
f_{k}(x)= \begin{cases}-k, & \text { if } 1 / k<x<2 / k \\ k, & \text { if } 2 / k<x<3 / k \\ 0, & \text { otherwise }\end{cases}
$$

The sequence $\left\{f_{k}\right\}$ converges pointwise to $f(x)=0$ on $[0,3]$ and is $\gamma$-convergent to $f$ on $[0,3]$. To see the latter, note that $\int_{0}^{1} f_{k}=0$ for each $k$. Consequently, for each positive integer $k$ there is a positive function $\delta_{k}$ defined on $[0,3]$ such that

$$
\left|S\left(f_{k}, P\right)-S(f, P)\right|=\left|S\left(f_{k}, P\right)-\int_{0}^{3} f_{k}\right|<\epsilon
$$

whenever $P$ is a $\delta_{k}$-fine tagged partition of $[0,3]$. However, this sequence is not uniformly Henstock integrable on $[0,3]$. Let $\delta$ be any positive function defined on $[0,3]$. Let $\delta(0)=\beta$ and choose a positive integer $n$ such that $2 / n<\beta$. Let $P_{1}$ be a $\delta$-fine tagged partition of $[2 / n, 3 / n]$ such that $S\left(f_{n}, P_{1}\right)>.9$ and let $P_{2}$ be a $\delta$-fine tagged partition of $[3 / n, 3]$. Then $P=(0,[0,2 / n]) \cup P_{1} \cup P_{2}$ is a $\delta$-fine tagged partition of $[0,3]$ and

$$
\left|S\left(f_{n}, P\right)-\int_{0}^{3} f_{n}\right|=S\left(f_{n}, P_{1}\right)>.9 .
$$

Therefore, the sequence $\left\{f_{k}\right\}$ is not uniformly Henstock integrable on $[0,3]$.

(As an aside, we point out that Bartle's Example 2(b) is not correct. The sequence $\left\{g_{k}\right\}$ defined in [1] can be shown to be uniformly Henstock integrable on $[0,2]$ and $\lim _{k \rightarrow \infty} \int_{0}^{2} g_{k}=0$. This follows from the facts that the supports of the $g_{k}$ 's are disjoint closed intervals. Note also that the indefinite integrals of the $g_{k}$ 's are equicontinuous on $[0,2]$.)

Now that we have examined the definitions, let's move on to a general convergence theorem. Since sequences are not sufficient to handle the limit process used in the definition of the Henstock integral, we must work with nets. In this case, it is best to be a little less general and work with the notion of a direction in a set. Here are the relevant definitions as found in McLeod [4]. 
Definition 2 Let $X$ be a nonempty set and let $f: X \rightarrow \mathbb{R}$.

(a) A direction $\Xi$ in $X$ is a nonempty collection of nonempty subsets of $X$ with the following property: for each pair of sets $S_{1}$ and $S_{2}$ in $\Xi$ there exists a set $S_{3} \in \Xi$ such that $S_{3} \subseteq S_{1} \cap S_{2}$.

(b) A real number $L$ is the limit of $f$ with respect to $\Xi$ if for each $\epsilon>0$ there exists a set $S_{\epsilon} \in \Xi$ such that $|f(x)-L|<\epsilon$ for all $x \in S_{\epsilon}$. This type of limit will be denoted by $L=\lim _{\Xi} f(x)$.

(c) The function $f$ is Cauchy with respect to $\Xi$ if for each $\epsilon>0$ there exists a set $S_{\epsilon} \in \Xi$ such that $\left|f\left(x_{1}\right)-f\left(x_{2}\right)\right|<\epsilon$ for all $x_{1}, x_{2} \in S_{\epsilon}$.

It is a standard exercise to prove that the limit of $f$ with respect to $\Xi$ is unique and that the limit of $f$ with respect to $\Xi$ exists if and only if $f$ is Cauchy with respect to $\Xi$. The key result is the following theorem on iterated limits. The main idea for this result was motivated by a theorem in Hobson [3, Section 305].

Theorem 2 Let $\Xi$ be a direction in $X$, let $\Upsilon$ be a direction in $Y$, and let $h: X \times Y \rightarrow \mathbb{R}$. Suppose that $f(x)=\lim _{\Upsilon} h(x, y)$ exists for each $x \in X$ and that $g(y)=\lim _{\Xi} h(x, y)$ exists for each $y \in Y$. Then the limits $\lim _{\Xi} f(x)$ and $\lim _{\Upsilon} g(y)$ both exist and are equal if and only if for each $\epsilon>0$ there exists a set $S_{\epsilon} \in \Xi$ such that for each $x \in S_{\epsilon}$ there exists a set $T_{x} \in \Upsilon$ such that $|h(x, y)-g(y)|<\epsilon$ for all $y \in T_{x}$.

Proof. Suppose first that the $\operatorname{limits} \lim _{\Xi} f(x)$ and $\lim _{\Upsilon} g(y)$ both exist and are equal and let $L$ be the common value of the limits. Let $\epsilon>0$. Since $\lim _{\Xi} f(x)=L$, there exists a set $S_{\epsilon} \in \Xi$ such that $|f(x)-L|<\epsilon / 3$ for all $x \in S_{\epsilon}$. Now fix $x_{0} \in S_{\epsilon}$. By hypothesis, there exist sets $T_{1}, T_{2} \in \Upsilon$ such that $\left|h\left(x_{0}, y\right)-f\left(x_{0}\right)\right|<\epsilon / 3$ for all $y \in T_{1}$ and $|g(y)-L|<\epsilon / 3$ for all $y \in T_{2}$. Choose $T_{x_{0}} \in \Upsilon$ such that $T_{x_{0}} \subseteq T_{1} \cap T_{2}$. For each $y \in T_{x_{0}}$,

$$
\begin{aligned}
\left|h\left(x_{0}, y\right)-g(y)\right| & \leq\left|h\left(x_{0}, y\right)-f\left(x_{0}\right)\right|+\left|f\left(x_{0}\right)-L\right|+|L-g(y)| \\
& <\epsilon / 3+\epsilon / 3+\epsilon / 3=\epsilon .
\end{aligned}
$$

Since $x_{0}$ was an arbitrary element of $S_{\epsilon}$, the first part of the proof is complete.

Now suppose that for each $\epsilon>0$ there exists a set $S_{\epsilon} \in \Xi$ such that for each $x \in S_{\epsilon}$ there exists a set $T_{x} \in \Upsilon$ such that $|h(x, y)-g(y)|<\epsilon$ for all $y \in T_{x}$. We first show that $f$ is Cauchy with respect to $\Xi$. Let $\epsilon>0$. By hypothesis, there exists a set $S_{\epsilon} \in \Xi$ such that for each $x \in S_{\epsilon}$ there exists a 
set $T_{x} \in \Upsilon$ such that $|h(x, y)-g(y)|<\epsilon$ for all $y \in T_{x}$. Let $x_{1}, x_{2} \in S_{\epsilon}$. Since $\lim _{\Upsilon} h\left(x_{1}, y\right)=f\left(x_{1}\right)$, there exists a set $T_{1} \in \Upsilon$ such that

$$
\left|h\left(x_{1}, y\right)-f\left(x_{1}\right)\right|<\epsilon \text { and }\left|h\left(x_{1}, y\right)-g(y)\right|<\epsilon
$$

for all $y \in T_{1}$. Similarly, there exists a set $T_{2} \in \Upsilon$ such that

$$
\left|h\left(x_{2}, y\right)-f\left(x_{2}\right)\right|<\epsilon \text { and }\left|h\left(x_{2}, y\right)-g(y)\right|<\epsilon
$$

for all $y \in T_{2}$. Choose $T \in \Upsilon$ such that $T \subseteq T_{1} \cap T_{2}$. For each $y \in T$,

$$
\begin{aligned}
\left|f\left(x_{1}\right)-f\left(x_{2}\right)\right| \leq & \left|f\left(x_{1}\right)-h\left(x_{1}, y\right)\right|+\left|h\left(x_{1}, y\right)-g(y)\right| \\
& +\left|g(y)-h\left(x_{2}, y\right)\right|+\left|h\left(x_{2}, y\right)-f\left(x_{2}\right)\right| \\
< & \epsilon+\epsilon+\epsilon+\epsilon=4 \epsilon .
\end{aligned}
$$

This shows that $f$ is Cauchy with respect to $\Xi$.

Let $L=\lim _{\Xi} f(x)$. We must show that $\lim _{\Upsilon} g(y)=L$. Let $\epsilon>0$. By hypothesis, there exists a set $S_{\epsilon} \in \Xi$ such that for each $x \in S_{\epsilon}$ there exists a set $T_{x} \in \Upsilon$ such that $|h(x, y)-g(y)|<\epsilon$ for all $y \in T_{x}$. In addition, there exists a set $S_{1} \in \Xi$ such that $|f(x)-L|<\epsilon$ for all $x \in S_{1}$. Choose $S \in \Xi$ such that $S \subseteq S_{\epsilon} \cap S_{1}$. Let $x_{0} \in S$. Since $\lim _{\Upsilon} h\left(x_{0}, y\right)=f\left(x_{0}\right)$, there exists a set $T_{1} \in \Upsilon$ such that $\left|h\left(x_{0}, y\right)-f\left(x_{0}\right)\right|<\epsilon$ for all $y \in T_{1}$. Choose $T \in \Upsilon$ such that $T \subseteq T_{1} \cap T_{x_{0}}$. Then

$$
\begin{aligned}
|g(y)-L| & \leq\left|g(y)-h\left(x_{0}, y\right)\right|+\left|h\left(x_{0}, y\right)-f\left(x_{0}\right)\right|+\left|f\left(x_{0}\right)-L\right| \\
& <\epsilon+\epsilon+\epsilon=3 \epsilon
\end{aligned}
$$

for all $y \in T$. It follows that $\lim _{\Upsilon} g(y)=L$. This completes the proof.

In order to apply this theorem on iterated limits to obtain a convergence theorem for the Henstock integral, we introduce the following notation. Let $X$ be the set of positive integers and let $Y$ be the set of all tagged partitions of $[a, b]$. Let $\Xi$ be the direction in $X$ defined by $\Xi=$ $\left\{S_{k}=\{k, k+1, \ldots\}: k \in X\right\}$ and let $\Upsilon$ be the direction in $Y$ defined by $\Upsilon=\left\{T_{\delta}: \delta\right.$ is a positive function on $\left.[a, b]\right\}$ where $T_{\delta}$ represents the set of all $\delta$-fine tagged partitions of $[a, b]$. Now let $\left\{f_{k}\right\}$ be a sequence of Henstock integrable functions defined on $[a, b]$ and suppose that $\left\{f_{k}\right\}$ converges pointwise to $f$ on $[a, b]$. Define $h: X \times Y \rightarrow \mathbb{R}$ by $h(k, P)=S\left(f_{k}, P\right)$. Note that

$$
\lim _{\Xi} h(k, P)=S(f, P) \quad \text { and } \quad \lim _{\Upsilon} h(k, P)=\int_{a}^{b} f_{k}
$$


for all $P \in Y$ and for all $k \in X$. If the resulting iterated limits exist and are equal, then

$$
\int_{a}^{b} f=\lim _{\Upsilon} S(f, P)=\lim _{\Xi} \int_{a}^{b} f_{k}=\lim _{k \rightarrow \infty} \int_{a}^{b} f_{k} .
$$

In other words, the function $f$ is Henstock integrable on $[a, b]$ and the value of its integral is the "expected" limit. Consequently, Theorem 2 (with the obvious analogue for $\Upsilon$ and $f$ ) leads to the following two theorems.

Theorem 3 Let $\left\{f_{k}\right\}$ be a sequence of Henstock integrable functions defined on $[a, b]$ and suppose that $\left\{f_{k}\right\}$ converges pointwise to $f$ on $[a, b]$. Then $f$ is Henstock integrable on $[a, b]$ and

$$
\int_{a}^{b} f=\lim _{k \rightarrow \infty} \int_{a}^{b} f_{k}
$$

if and only if for each $\epsilon>0$ there exists a positive integer $N_{\epsilon}$ such that for each $k \geq N_{\epsilon}$ there exists a positive function $\delta_{k}$ defined on $[a, b]$ such that $\left|S\left(f_{k}, P\right)-S(f, P)\right|<\epsilon$ for all $\delta_{k}$-fine tagged partitions of $[a, b]$.

Corollary 4 Let $\left\{f_{k}\right\}$ be a sequence of Henstock integrable functions defined on $[a, b]$. If $\left\{f_{k}\right\}$ converges uniformly to $f$ on $[a, b]$, then $f$ is Henstock integrable on $[a, b]$ and

$$
\int_{a}^{b} f=\lim _{k \rightarrow \infty} \int_{a}^{b} f_{k}
$$

Theorem 5 Let $\left\{f_{k}\right\}$ be a sequence of Henstock integrable functions defined on $[a, b]$ and suppose that $\left\{f_{k}\right\}$ converges pointwise to $f$ on $[a, b]$. Then $f$ is Henstock integrable on $[a, b]$ and

$$
\int_{a}^{b} f=\lim _{k \rightarrow \infty} \int_{a}^{b} f_{k}
$$

if and only if for each $\epsilon>0$ there exists a positive function $\delta$ defined on $[a, b]$ such that for each $\delta$-fine tagged partition $P$ of $[a, b]$ there exists a positive integer $N_{P}$ such that $\left|S\left(f_{k}, P\right)-\int_{a}^{b} f_{k}\right|<\epsilon$ for all $k \geq N_{P}$.

Corollary 6 Let $\left\{f_{k}\right\}$ be a sequence of Henstock integrable functions defined on $[a, b]$ and suppose that $\left\{f_{k}\right\}$ converges pointwise to $f$ on $[a, b]$. If $\left\{f_{k}\right\}$ is uniformly Henstock integrable on $[a, b]$, then $f$ is Henstock integrable on $[a, b]$ and

$$
\int_{a}^{b} f=\lim _{k \rightarrow \infty} \int_{a}^{b} f_{k}
$$


Theorem 3, with pointwise convergence added to the definition of $\gamma$-convergence, is the result proved by Bartle. As indicated by the corollary, this theorem represents some sort of weakening of uniform convergence of $\left\{f_{k}\right\}$ to $f$. Theorem 5 gives a different perspective on the convergence theorem. It is more in the spirit of uniform Henstock integrability.

This paper was inspired by the reading of a preprint of an article by Isidore Fleischer. His paper, "The convergence content of the integral limit interchange theorem", also appears in this volume of the Real Analysis Exchange.

\section{References}

[1] R. G. Bartle, A convergence theorem for generalized Riemann integrals, Real Analysis Exchange, 20 (1994-95), 119-124.

[2] R. A. Gordon, Another look at a convergence theorem for the Henstock integral, Real Analysis Exchange, 15 (1989-90), 724-728.

[3] E. W. Hobson, The theory of functions of a real variable and the theory of Fourier series, vol. 1, 3rd ed., Cambridge, 1927.

[4] R. M. McLeod, The generalized Riemann integral, Carus Mathematical Monographs, no. 20, Mathematical Association of America, 1980. 